\title{
Significado, manifestaciones, actores y causas de la violencia desde la mirada de los jóvenes
}

\section{Meaning, manifestations, actors, and causes of violence from young people's perspective}

Lourdes Cortés Ayala

Mirta Flores Galaz

Teresa Morales Manrique

Universidad Autónoma de Yucatán

\section{Resumen}

La presente investigación tuvo como objetivo realizar un análisis de las diferencias en la percepción que tienen hombres y mujeres de la ciudad de Mérida acerca del significado de la violencia y sus diferentes tipos, sus causas, consecuencias, y posibilidades de prevención, así como las características de los actores, de víctimas y agresores. Participaron 22 personas, originarias de la ciudad de Mérida, Yucatán, que fueron seleccionadas mediante un muestreo no probabilístico; 10 hombres y 12 mujeres, con una edad promedio de 22.45 años y una desviación estándar de 2.32. Se realizó una entrevista estructurada, conformada por 22 preguntas que exploraban los conocimientos y creencias sobre la violencia interpersonal: a) significado (caracterización, tipos, escenarios); b) agresores (características, lugares en que ocurre, causas para agredir, posibilidad de ayudarlos); c) víctimas (características, cómo ayudar a víctimas) y d) causas de la violencia, consecuencias y prevención (cómo prevenir, qué medidas conoces). Los hallazgos evidencian conocimientos limitados sobre la violencia interpersonal, aunque se conocen diferentes expresiones de violencia y algunos contextos en los que éstas se manifiestan. Asimismo, se observa que existen mitos sobre la causa de la violencia y las características de los agresores y víctimas, así como sobre la posibilidad de prevenirla. Se concluye que es necesario promover el conocimiento para reforzar y sensibilizar a través de diferentes estrategias a los jóvenes con el fin de prevenir cualquier tipo de violencia.

Palabras clave: violencia, agresores, víctimas, jóvenes, prevención.

Nota del autor

Lourdes Cortés Ayala, Mirta Flores Galaz y María Teresa Morales Manrique, Facultad de Psicología, Universidad Autónoma de Yucatán.

La correspondencia en relación a este artículo debe dirigirse a Lourdes Cortés Ayala, Facultad de Psicología, Campus de Ciencias Sociales, Carretera Tizimín-Cholul Km 1. CP 97305, Mérida, Yucatán. Correo electrónico: lourdes.cortes@correo.uady.mx 


\begin{abstract}
The primary objective of this research project was to analyze differences in the perceptions of men and women in the city of Mérida regarding the meanings of violence and its types, the characteristics of the actors, victims, and aggressors, as well as its causes, consequences, and possibilities for prevention. Twenty-two people were selected through a non-probabilistic sampling of the city of Mérida, Yucatán: ten men and 12 women, with an average age of 22.45 years and a standard deviation of 2.32. Structured interviews consisting of 22 questions that explored knowledge and beliefs about interpersonal violence were carried out: a) meaning (characterization, types, scenarios); b) aggressors (characteristics, places where it occurs, causes, possibility of preventing them); c) victims (characteristics, how to help victims); and d) causes of violence, consequences, and prevention (how to prevent, what preventative measures are known). The findings showed limited knowledge about interpersonal violence, although they know different expressions of violence and some contexts in which it is manifested. Likewise, it was observed that there are widely-held stereotypes about the causes of violence and the characteristics of the aggressors and victims, as well as about the possibility of preventing it. We conclude that it is necessary to promote knowledge in order to reinforce and sensitize young people through different strategies in an effort to prevent any type of violence.
\end{abstract}

Keywords: violence, aggressors, victims, youth, prevention.

En la realidad cotidiana, la violencia tiene un importante protagonismo, expresado en múltiples formas. Aunque aparece como una constante en la historia, toma formas singulares y específicas; se objetiviza de maneras diversas y asume en cada sociedad un papel específico (García-Canal, 2006). En el mundo actual, la violencia es vivida por innumerables personas y destroza la vida de millones de ellas, como señaló Mandela (Organización Panamericana de la Salud, 2002); se vive desde "el sufrimiento invisible de los más vulnerables de la sociedad a la tragedia notoria de las sociedades en conflicto" (p. v). En el último Informe sobre la situacion mundial de Violencia (World Health Organization, 2016), se señala que en todo el mundo casi medio millón de personas son asesinadas cada año.

La violencia en sus múltiples formas se ha considerado un fenómeno inevitable en la vida cotidiana, ante el cual únicamente se puede reaccionar. Sin embargo, a partir del Informe Mundial sobre Violencia y Salud (OMS, 2002), se presentó el panorama de esta problemática a escala mundial, y se evidenció su naturaleza aprendida y sus diversas facetas, los ámbitos donde se desarrolla y la posibilidad ineludible de prevenirla. La Organización Mundial de la Salud (OMS, 2002, p. 3), define la violencia como:

El uso deliberado de la fuerza física o el poder, ya sea en grado de amenaza o 
efectivo, contra uno mismo, otra persona o un grupo o comunidad que cause o tenga muchas probabilidades de causar lesiones, muerte, daños psicológicos, trastornos del desarrollo o privaciones.

Esta definición también incluye a la violencia interpersonal, el comportamiento suicida $\mathrm{y}$ los conflictos armados; a esta definición se suman las amenazas e intimidaciones, así como las consecuencias menos evidentes del comportamiento violento: daños psíquicos, privaciones y deficiencias del desarrollo que comprometen el bienestar de los individuos, las familias y las comunidades (OMS, 2002, p. 3). Algunas expresiones de la violencia son más visibles que otras, por ejemplo, la violencia comunitaria y la juvenil, que constituyen un delito, en tanto la violencia intrafamiliar (maltrato a menores y ancianos o violencia a la esposa) está mucho más oculta. La OMS (2016) refiere que las mujeres, los niños y las personas mayores son quienes reciben mayor violencia de tipo físico, psicológico y sexual no mortales. La violencia puede ser clasificada como autoinflingida, interpersonal y colectiva y éstas, a su vez, pueden tener consecuencias mortales o no.

La violencia es considerada un problema de salud pública, ya que gran parte del costo que implica vivir con violencia repercute en la salud de las víctimas y en los costos del sistema de salud de los países (Miller, Cohen \& Rossman, 1993; OMS, 2016). Asimismo, constituye un fenómeno de causalidad compleja, sobredeterminado tanto por lo intrapsíquico como por lo social (Foucalt, 1999). Jiménez-Bautista (2012) señala que "el ser humano es conflictivo por naturaleza, pero pacífico o violento por cultura... la violencia está en su ambiente" (p. 14). El conflicto es un fenómeno connatural a la existencia humana que se presenta en diversas interacciones y relaciones sociales (Silva, 2008; Maliandi, 2010; Pegalajar, 2018), surge cuando "un actor percibe que uno a varios de sus fines, preferencias o medios para alcanzarlos es amenazado o estorbado por las intenciones o actividades de uno o varios actores con que interactúa, lo que conduce a una oposición, enfrentamiento o lucha" (Osorio, 2012, p. 64). La resolución de conflictos interpersonales es parte de la vida misma, sin embargo, esta resolución muchas veces se realiza a través de la violencia aprendida por los individuos en su entorno psicosocial: tal entorno estrechamente ligado a la expresión de poder, incluso se ha conceptuado como la expresión más contundente del poder (Arendt, 2005). El poder es el ejercicio de unos sobre las acciones de otros; entendido desde una óptica más actual, se lo considera un potencial humano para hacer algo, conseguir objetivos específicos e intereses personales, y para obtener de una situación un resultado favorable a los propios intereses (Esquivel, Jiménez-Bautista \& EsquivelSánchez, 2009).

Para explicar la violencia y trabajar en su prevención, la OMS ha propuesto, desde el año 2002, el modelo ecológico, pues considera que 
no existe un factor por sí solo, apto para explicar por qué una persona se comporta violentamente. Este modelo está conformado por cuatro niveles: individual, relacional, comunitario y social. Los factores individuales incluyen variables biológicas, la historia personal, características demográficas, trastornos psíquicos o de personalidad, toxicomanías y antecedentes de maltrato. Los factores a nivel relacional se refieren a las experiencias de relación con otros, como haber sufrido castigos severos durante la infancia, falta de afecto y de vínculos emocionales, entre otros. Los comunitarios y sociales tienen que ver con la exposición a la violencia a través de los medios de comunicación, vivir en barrios con altas tazas de delincuencia, pobreza, o bien, lugares con políticas de protección social débiles (Quiroga \& Cryan, 2007; Unnithan \& Whitt, 1992).

Sobre su incidencia, en las personas de 15 a 44 años, la violencia es la cuarta causa de muerte a escala mundial (WHO, Global Health Observatory, 2016. Decenas de miles de personas en todo el mundo son víctimas de la violencia no mortal a diario; sufren lesiones físicas, maltrato psicológico y abuso sexual.

En México, las diversas manifestaciones de violencia se han incrementado en los últimos años; las estadísticas ubican el 2017 como el año más violento en cuanto a la cifra de homicidios $\mathrm{y}$ de feminicidios, incrementándose este último en $18 \%$ respecto al año anterior (INEGI, 2017). Violencia de género, violencia feminicida, bullying o violencia en la escuela, mobbing o acoso laboral, acoso callejero, son algunas de las manifestaciones de violencia que se viven cotidianamente y que se han incrementado. Como muestra de ello, los estudios a nivel nacional evidencian que la violencia contra las mujeres de 15 años o más ha ido en aumento durante los últimos 10 años, y se reporta que $66.1 \%$ ha enfrentado al menos un incidente en su vida. Asimismo, esta violencia ocurre en todos los ámbitos de convivencia, tanto públicos (escuelas, lugares de trabajo, comunidad) como privados (el hogar) (ENDIREH, 2016). Además, los datos del Sistema Nacional de Seguridad Pública (SNSP), indicaron que al cierre de 2017 se incrementó el número de homicidios intencionales en $27 \%$ respecto a 2016, se reportó en el país una violencia sin precedente con 80 asesinatos al día. Otras investigaciones muestran que cuatro de cada 100 jóvenes tienen problemas de salud debido a la violencia interpersonal vivida, donde la prevalencia de esta violencia es mayor en hombres (5\%) que en mujeres $(3.3 \%)$, además, el grupo de hombres jóvenes (20-29 años) se constituye como el grupo más vulnerable (Valdez-Santiago, Hidalgo-Solórzano, Mojarro-Íñiguez, RiveraRivera \& Ramos-Lira, 2013).

Frías y Castro (2011) señalan que ante esta problemática se han realizado múltiples investigaciones en nuestro país con el propósito de identificar los determinantes sociales de diversas formas de violencia en distintos ámbitos (familiar, escolar, de pareja), sobre todo desde la perspectiva clínica y psicológica, de salud 
pública y de corte demográfico; sin embargo, los autores consideran que la investigación respecto a la violencia interpersonal a lo largo de la vida del individuo, de corte sociológico, es escasa. En esta línea, los resultados de varias investigaciones desde la psicología, han mostrado que la perpetración de violencia hacia los hijos o la pareja (cónyuge/novio) es producto, en gran parte, de la socialización hacia la violencia en el hogar familiar (Cerezo \& Ato, 2010; Fuentes, Martínez \& Navarro, 2015; Suárez, Del Moral, Martínez \& Musitu, 2015; Romero-Abrio, Musitu, Callejas-Jerónimo, Sánchez-Sosa \& Villarreal-González, 2018). Otros estudios muestran los efectos de variables contextuales como pobreza, alcohol, e incluso la falta de legislación que proteja a niños y/o mujeres (Freisthler, Merritt \& LaScala, 2006; Gracia \& Herrero, 2008; Molnar, Buka, Brennan, Holton \& Earls, 2003), y de variables de personalidad, como baja autoestima, depresión, adicción a sustancias (Oliver, Kuhns \& Pomeranz, 2006; Tajima, 2002), entre las principales. También la transmisión intergeneracional de la violencia ha sido ampliamente documentada, sobre todo en lo relativo a violencia contra las mujeres y las niñas (Terceño, 2017).

La socialización en la violencia, es decir, en contextos violentos desde la infancia, constituye una variable crítica en la perpetración de violencia y en el sufrimiento de la misma. Un amplio estudio realizado por Frías y Castro (2011), con abordaje de la trayectoria de vida (desde primera infancia a la crianza de los hijos, pasando por la vida escolar, el noviazgo y la vida en pareja), ha evidenciado que la existencia de violencia física en cada etapa incrementa las probabilidades de que exista violencia en las siguientes etapas; otra variable relevante, además de la socialización en la violencia, es la exposición directa o indirecta a formas violentas de resolución de conflictos en ámbitos familiares, escolares e incluso del vecindario del individuo.

La violencia interpersonal en México afecta a individuos, instituciones y sociedad en general, con serios efectos negativos en la salud, economía y política, así como en el bienestar psicológico y social de los individuos (BriceñoLeón, 2008; Valdez-Santiago et al., 2013). Un estudio realizado en Monterrey por Rubio, Chávez y Rodríguez (2017) muestra que los jóvenes reconocen diversos tipos de violencia, así como sus causas (adicciones, desintegración familiar y diversos efectos a nivel psicológico individual, familiar, en su círculo de amigos y comunidad). García Canal (2006), afirma que la palabra violencia alude a una experiencia de todos los días y se vincula con diversas ideas como agresión y agresividad, daño, coerción, violación, robo, estupro, dolo, herida, herida del cuerpo, dolo e incluso asesinato y muerte.

En Yucatán, una revisión de los diversos estudios sobre violencia de género, realizada por Paredes-Guerrero, Llanes-Salazar, Torres-Salas y España Paredes (2016), concluye que sólo se contempla la dimensión interpersonal o directa de la violencia ocurrida en el espacio privado 
del hogar, dejando de lado la violencia ocurrida en el espacio público, institucional y feminicida, lo cual limita la explicación del fenómeno en la entidad. Asimismo, la necesidad de generar conocimiento propio en el contexto de la ciudad de Mérida respecto a la conceptuación de la violencia, para ulteriormente trabajar en acciones encaminadas a la prevención de la misma, guía el interés de este trabajo sobre cómo perciben la violencia los jóvenes. Por ello, el presente estudio tiene como objetivos:

1. Realizar un estudio exploratorio sobre la percepción que tienen hombres y mujeres de la ciudad de Mérida acerca del significado de la violencia y sus tipos, características de los actores, de víctimas y agresores, las causas, consecuencias y posibilidades de prevención.

2. Describir las diferencias en la percepción que tienen hombres y mujeres de la ciudad de Mérida sobre el significado de la violencia y sus tipos, características de los actores, de víctimas y agresores, las causas, consecuencias y posibilidades de prevención.

\section{Participantes}

\section{Método}

Participaron 22 personas, 10 hombres y 12 mujeres, con un rango de edad de 20 a 30 años, con una edad promedio de 22.45 años y una desviación estándar de 2.32; en su mayoría, eran estudiantes de bachillerato y de universidad de diferentes carreras (Enfermería, Ingeniería,
Administración, entre otras), una minoría estudiaba y trabajaba.

\section{Instrumento}

Se realizó una entrevista con el objetivo de explorar los conocimientos y creencias sobre la violencia interpersonal. El guion de la entrevista se conformó por 22 preguntas elaboradas para explorar las siguientes categorías o dimensiones: a) significado (caracterización, tipos, escenarios); b) agresores (características, lugares en que ocurre, causas para agredir, posibilidad de ayudarlos); c) víctimas (características, cómo ayudar a víctimas) y d) causas de la violencia, consecuencias y prevención (cómo prevenir, qué medidas conoces).

\section{Procedimiento}

Los participantes respondieron en forma individual a la entrevista, previa explicación de los objetivos del estudio, y haber firmado el consentimiento informado que les garantizaba anonimato y confidencialidad de sus respuestas. Las entrevistas fueron realizadas por seis entrevistadores en los lugares elegidos por los participantes (su domicilio, escuela o un parque); todas las entrevistas fueron grabadas y transcritas para su posterior análisis de contenido (Hernández-Sampieri, FernándezCollado \& Baptista-Lucio, 2014). Se analizaron las respuestas dadas, para cada categoría se identificaron las subcategorías y para éstas los conceptos relevantes (códigos); posteriormente, también se obtuvieron las frecuencias de los 
códigos, tanto para hombres como para mujeres, en cada una de las categorías exploradas (significado, agresores, víctimas, causas de la violencia, consecuencias y prevención).

\section{Dimensión significado}

\section{Resultados}

Al indagar el significado que le atribuyeron al término violencia (¿qué es violencia?), los participantes dieron respuestas que se orientan en tres dimensiones: hacia quien va dirigida, el escenario donde ocurre, o bien, los tipos de violencia conocida (ver tabla 1). El análisis de las frecuencias de las diversas respuestas muestra que cerca de la mitad de los participantes la definió como una "agresión o abuso" y añadieron que puede ser "de tipo físico o mental". Pocas mujeres consideraron que es algo malo (o maligno) y que produce daño. Asimismo, se reconocieron diversos tipos de violencia, aunque todos los participantes mencionaron la violencia física en primer término, a la cual identificaron con "golpes". También mencionaron la violencia verbal (realizada a través de ofensas), la violencia sentimental, emocional o psicológica. A la descripción sobre qué es violencia, se añadió la noción de poder, ya que mencionaron el hecho de "someter a alguien para que haga algo que quiera". Llama la atención que únicamente una participante mencionó la violencia familiar como contexto de manifestación de esta problemática. Dos varones señalaron, además, que la violencia puede ser voluntaria e intencional o involuntaria. Otros dos comentaron sobre la naturaleza de la violencia, refiriendo que puede ser aprendida de la cultura, o bien, que alude a acciones no aprobadas por la sociedad e, incluso, se mencionó que puede ser algo innato o intencional. Una chica indicó que puede justificarse: "pues a veces tiene motivos, pero a veces no hay motivos". Cuatro participantes mujeres enfatizaron que la violencia supone atentar contra la integridad, por lo cual conlleva degradar, denigrar o hacer sentir mal o hacer sufrir a otro/a.

Para abundar en este tema, más tarde se les hizo la pregunta “¿qué es para ti un acto violento?". En este caso, fueron las mujeres quienes aludieron más a los golpes o al uso de la fuerza física, añadiendo la violencia verbal, como lo insultos y ofensas: "si yo vengo y te insulto... no te golpeo, pero te estoy dañando". Mencionaron también la violencia psicológica, pero no proporcionaron ejemplos de este tipo. Por último, los participantes incluyeron aquellas acciones que hacen daño, y atentan contra la libertad de otro intencionalmente; aunque aquí fueron los hombres quienes elaboraron más este tipo de argumentos, en tanto que las mujeres citaron como tipos de conducta violenta las agresiones, insultos, imposiciones de forma de pensar y limitaciones a la libertad, por ejemplo, una chica mencionó: “Que no te dejen salir”.

Respecto a los tipos o formas de violencia, las mujeres reconocieron quince tipos de manifestación de violencia hacia otra persona, mientras que los hombres mencionaron nueve. 
Tabla 1

Categorías de respuestas de hombres y mujeres ante la pregunta, ¿qué es violencia?

\begin{tabular}{|c|c|c|}
\hline Dirigida a: & Mujeres & Hombres \\
\hline Mujeres & $\begin{array}{l}\text { "Menospreciar a las mujeres, que le niegan sus } \\
\text { derechos a una persona", "que por ser mujer te } \\
\text { hagan menos" }\end{array}$ & Dañar por su género o sexo \\
\hline $\begin{array}{l}\text { Personas con } \\
\text { discapacidad }\end{array}$ & $\begin{array}{l}\text { "Hay personas que le dicen a los discapacitados, } \\
\text { eres un lisiado o quítate" }\end{array}$ & \\
\hline Animales & $\begin{array}{l}\text { "Cuando le pegan a un perro, los matan o abandonan } \\
\text { sin motivo" }\end{array}$ & \\
\hline \multicolumn{3}{|l|}{ Escenario } \\
\hline $\begin{array}{l}\text { Bullying o } \\
\text { Violencia } \\
\text { Escolar }\end{array}$ & $\begin{array}{l}\text { Insultos, hacen valer menos a la otra persona y } \\
\text { golpes, "grupito de niños agarra a un niño y siempre } \\
\text { lo friegan" }\end{array}$ & $\begin{array}{l}\text { Insultar, golpes, privación de } \\
\text { necesidades básicas, como } \\
\text { comida, agua }\end{array}$ \\
\hline $\begin{array}{l}\text { Mobbing o } \\
\text { Violencia } \\
\text { Laboral }\end{array}$ & "Exceda de tiempo de los trabajadores" & $\begin{array}{l}\text { Paguen menos por ser mujer, } \\
\text { discriminación, tratar mal, daño } \\
\text { psicológico, daño verbal, abuso } \\
\text { del patrón }\end{array}$ \\
\hline $\begin{array}{l}\text { Violencia } \\
\text { intrafamiliar }\end{array}$ & Golpes en la familia, insultos y golpes & \\
\hline $\begin{array}{l}\text { Violencia } \\
\text { Virtual }\end{array}$ & Molestar o acosar por redes sociales & Ciberacoso \\
\hline
\end{tabular}

\section{Tipo}

\begin{tabular}{|c|c|c|}
\hline $\begin{array}{l}\text { Violencia } \\
\text { Física }\end{array}$ & $\begin{array}{l}\text { Golpes, jalones de pelo pellizcos, moretones, } \\
\text { pegar, es muy obvio }\end{array}$ & $\begin{array}{l}\text { Golpes, empujones o lastimar con } \\
\text { algún otro objeto }\end{array}$ \\
\hline $\begin{array}{l}\text { Violencia } \\
\text { Verbal }\end{array}$ & $\begin{array}{l}\text { Insultos, agresiones verbales y decir cosas } \\
\text { desagradables, ofensas, humillaciones, palabas } \\
\text { altisonantes que te hieren o te hacen sentir menos }\end{array}$ & $\begin{array}{l}\text { Decir cosas de forma hiriente } \\
\text { para hacer daño, relación con la } \\
\text { violencia psicológica }\end{array}$ \\
\hline $\begin{array}{l}\text { Violencia } \\
\text { Sexual }\end{array}$ & $\begin{array}{l}\text { Que te obliguen a hacer cosas que no quieres como } \\
\text { tener relaciones sexuales, violaciones, acosar } \\
\text { sexualmente a una mujer }\end{array}$ & $\begin{array}{l}\text { Acoso, extorsión, violaciones, } \\
\text { filtrar fotos, atentar contra tus } \\
\text { derechos sexuales }\end{array}$ \\
\hline $\begin{array}{l}\text { Violencia } \\
\text { Psicológica }\end{array}$ & $\begin{array}{l}\text { Por medio de las palabras como "estás gorda," } \\
\text { dañan su mente y sus emociones. Son los insultos } \\
\text { que te dicen... tiene que ver con actos verbales y } \\
\text { autoestima. Hacen que las víctimas tengan más } \\
\text { estados emocionales negativos que positivos }\end{array}$ & $\begin{array}{l}\text { Daño verbal a otros, por control, } \\
\text { manipulación, ataque a las } \\
\text { emociones, autoestima y chantaje }\end{array}$ \\
\hline $\begin{array}{l}\text { Violencia } \\
\text { Emocional }\end{array}$ & Intimidar a personas & Daño a la autoestima, control \\
\hline $\begin{array}{l}\text { Violencia } \\
\text { Económica }\end{array}$ & & $\begin{array}{l}\text { Quitar o no dar dinero, bienes } \\
\text { materiales }\end{array}$ \\
\hline
\end{tabular}


Las respuestas se pueden clasificar en tipos de violencia (física, verbal, psicológica, sexual, de género, emocional, económica), contextos de expresión (familiar, laboral, pareja, virtual, calle, bullying) y víctimas (personas con discapacidad, animales, niños). Se evidencia nuevamente que la violencia física fue la más reconocida y mencionada en primer lugar por ambos, seguida de la violencia verbal y psicológica. Mayor número de mujeres que de hombres reconoció la violencia sexual; además, los hombres no mencionaron la violencia de pareja, mientras que las mujeres sí lo hicieron.

En cuanto a los escenarios donde se cree pueda ocurrir la violencia, la mayoría (20 de 21 participantes) considera que la violencia se puede presentar en todos los escenarios; un comentario al respecto es el siguiente: "no veo un ambiente que pueda estar libre de ella... al final yo creo que podemos decir que puede haber violencia donde haya personas". Al explorar todos los posibles escenarios, más de la mitad de las mujeres mencionó el espacio privado del hogar como escenario primordial de violencia, empero, para la mayoría de los hombres la violencia ocurre, sobre todo, en el espacio laboral. En términos de contextos de relación, las mujeres señalaron cuatro propicios para la violencia: matrimonio, noviazgo, amigos, familiar; mientras que los hombres solamente consideraron dos: el familiar y con amistades.

\section{Dimensión agresores}

Sobre las características que distinguen a una persona violenta, hombres y mujeres coincidieron en que quienes ejercen violencia son personas con ciertas características de personalidad, en especial, agresividad, impulsividad, enojo y manipulación; tienen autoestima baja y están reprimidass. En menor proporción, ambos señalaron que pueden tener una disfunción mental que les lleve a ser poco pacientes e inestables mentalmente. Muchas más mujeres que hombres señalaron que no puede identificarse físicamente a una persona violenta, pues parecen "normales", por consiguiente, cualquier persona puede ser violenta.

Respecto a los lugares en los que han presenciado o vivido un acto violento, hombres $\mathrm{y}$ mujeres describieron lugares públicos o concurridos como bares, antros, fiestas, reuniones, colonias, parques, canchas o plazas, al igual que en la casa y en la escuela; añadieron que puede ocurrir en la prepa, secundaria o universidad. Las mujeres mencionaron el internet/redes sociales, así como el contexto de la relación de noviazgo o en la pareja, a diferencia de los hombres, quienes no mencionaron estos contextos de relación. Únicamente los hombres respondieron haber presenciado o vivido un acto violento en el contexto laboral.

En torno a ¿por qué se cree que una persona ejerce violencia (de cualquier tipo) contra otra?, las respuestas de hombres y mujeres hicieron referencia a factores de personalidad (baja 
autoestima o falta de seguridad, emociones negativas, sentimiento de superioridad, falta de control de impulsos, temperamento e incluso mencionaron que por placer o deseo). Como factores contextuales, señalaron la influencia o aprendizaje en la familia, el haber aprendido a comportarse violentamente, y también por biología, ya que algunos son más violentos que otros. Para las mujeres, la familia fue el factor más importante, al señalar que se debe a conductas aprendidas desde la infancia en su ambiente familiar, por ejemplo, una participante señaló: “creo que esa persona en algún momento fue dañada o creció en un lugar violento y lo que aprendió lo está aplicando con los demás”. También las mujeres atribuyeron la violencia a la baja autoestima o falta de seguridad y la necesidad de ejercer poder o sentirse superiores. En contraste, los hombres consideraron, con mayor frecuencia, que la causa principal es la falta de control de impulsos, así como la necesidad de ejercer poder y sentirse superior, al señalar que la persona agresiva no sabe cómo manejar sus emociones, así que las canaliza de una manera negativa hacia una persona de manera violenta, por ejemplo, un participante comentó: "Bueno pues ahí ya sería más circunstancial, en el acto, pues a lo mejor es una frustración mal gestionada, un impulso, una provocación y ya sea premeditada por su parte o podría ser provocación de alguien más con la intención de que esa persona sea un victimario".

Sobre la posibilidad de brindar ayuda a los agresores, todas las personas respondieron afirmativamente. Cuándo se cuestionó específicamente sobre ¿cómo se podría ayudar a los agresores?, la gran mayoría de hombres y mujeres coincidió en que la ayuda psicológica es el medio más adecuado para los agresores, y agregó que las pláticas, o bien, promover la comunicación con ellos ayudaría a esta problemática, y señaló que podría ser a través de recomendarles y/o llevar a la persona violenta a instituciones para que reciba ayuda psicológica o pláticas de autoayuda. Otras acciones propuestas fueron brindar apoyo al agresor, hacer algo ante una situación violenta, informar a una autoridad, darle seguimiento a la persona agresora, trabajar en su ambiente y recomendar actividades que ayuden a su salud emocional. Ambos sexos mostraron empatía hacia la persona agresora, al tratar de entender las posibles razones que la llevan a cometer los actos violentos, sin embargo, algunos participantes declararon no saber qué hacer para ayudarla.

\section{Dimensión víctimas}

La mayoría de los participantes consideró que las víctimas de violencia son aquellas personas que sufren o reciben maltrato, acoso, golpes o agresiones, y enfatizó que cualquiera puede ser víctima de violencia. Sin embargo, atribuyó una serie de características de personalidad a las víctimas, al indicar que son personas pasivas y sumisas; las mujeres señalaron que las víctimas tienen problemas psicológicos. Algunos hombres consideraron que las víctimas pueden ser culpables de provocar la violencia: 
"sería la receptiva, la que podría decirse que por su culpa o que lo haya provocado... si esa es la persona afectada", o malinterpretar una situación como violencia, "la persona no lo dice con la intención de agredir, pero una persona si se siente agredida, entonces igual podría ser una víctima". Ambos indicaron que la característica de personalidad de víctimas de violencia son limitaciones en habilidades sociales, como señala un participante: "puede ser cualquier persona... pero puede ser... no sé, desde mi opinión muy personal, puede ser... una persona que sea muy susceptible a que la puedan ver débil, que la puedan ver callada". Las mujeres, a diferencia de los hombres, mencionaron que sobre todo mujeres, ancianos e incluso los animales igual podrían ser víctimas de violencia. Los hombres señalaron como posibles víctimas a personas de escasos recursos, homosexuales y unos pocos consideraron a los mismos hombres.

Cuando se abordó el tema de ¿cómo creen que puede ayudarse a las víctimas de violencia?, las respuestas pueden clasificarse a partir de dos categorías: ayuda dirigida a la persona y ayuda indirecta o proveniente de alguna instancia pública, o bien, acciones colectivas contra la problemática. Asimismo, las respuestas hacen referencia a ofrecer ayuda al agresor. Hombres y mujeres coincidieron en que es importante "defender a las víctimas", "alzar la voz ante una autoridad" o "apoyarlas en el momento que están viviendo a través de acciones como marchas", así como "crear leyes que castiguen al agresor". Añadieron que la ayuda individual consistiría en ofrecer el apoyo legal a víctimas, así como ayuda psicológica al agresor. Los hombres respondieron que acciones como orientar, instruir, platicar y escuchar acerca del problema ayuda a las víctimas. Las mujeres, a diferencia de los hombres, mencionaron la importancia de promover o generar conciencia sobre el tema, $y$ los hombres coincidieron en que las campañas de prevención, talleres y conferencias serían de ayuda.

Otras respuestas ponen de manifiesto que las mujeres tendieron a tomar una postura menos directa de ayuda frente a una situación de violencia: "no meterse... porque si yo me meto a defenderla, me puede ir peor y van a ser dos violentadas", siendo la alternativa esperar y llamar a alguna autoridad que pueda ayudar. En contraste, los hombres entrevistados sí mencionaron la importancia de intervenir de manera directa ante un acto violento, teniendo contacto con el agresor, separando o defendiendo a la víctima.

\section{Dimensión causas de la violencia, consecuencias, y prevención}

\section{a) Causas de la violencia.}

Respecto a lo que consideran las causas o lo que ocasiona la violencia, los participantes aludieron con más frecuencia a factores relacionados con la familia y los estilos de crianza, así como a la construcción social de la violencia debido a los aprendizajes del contexto y ambiente. También refirieron las necesidades fisiológicas (de los hombres), el machismo, la falta de habilidades de comunicación, el abuso 
de poder y las experiencias de aprendizaje pasadas. Para las mujeres, la familia es el factor más señalado como causante de violencia, que inicia generalmente con un seno familiar violento o carente de cariño y afecto para el infante en desarrollo, también se refirieron al ambiente violento a través de todo ciclo vital en la familia. Se hace alusión a cómo el entorno afecta al desarrollo conductual, al enseñar a ser violento/a. Los hombres con frecuencia señalaron los sucesos del pasado, las situaciones de la vida y las experiencias de los primeros años en el entorno familiar: "que te pueden llevar a ocasionar violencia como una consecuencia directa de estas situaciones, donde se aprende el machismo".

Al preguntar ¿qué consideras que vuelve a las personas violentas? En general, las respuestas de los participantes apuntan hacia tres tipos de factores causales: psicológicos, sociales y biológicos. Los factores psicológicos aluden a características emocionales y cognitivas del agresor, a saber, la falta de regulación de emociones como el enojo o el estrés, además de poseer rasgos de personalidad como la agresividad y la impulsividad. Los factores sociales hacen referencia al machismo, la cultura, las relaciones de poder, la desigualdad y el ambiente social. Un participante mencionó el papel de la estructura cerebral y neurotransmisores en la manifestación de la violencia y otro refirió el tráfico de drogas y el consumo de sustancias como factor causante de violencia.
En lo relativo a ¿por qué una persona permite que ejerzan violencia contra ella?, las respuestas pueden agruparse también en dos categorías: psicológicas $\mathrm{y}$ factores sociales. Como características psicológicas de la víctima se mencionaron el miedo a ser agredidas, amenazadas o intimidadas, baja autoestima (sentimiento de inferioridad y de no valer nada); y entre los factores sociales se contaron la falta de redes de apoyo, desigualdad económica, las experiencias de la crianza, características del contexto donde ocurre, relaciones de poder o superioridad. Las mujeres con más frecuencia subrayaron el papel de los factores psicológicos, asi como la normalización de la violencia como forma de comportarse. Los hombres señalaron que ocurre porque la víctima no sabe cómo defenderse. En cuanto a factores sociales, indicaron que las situaciones de poder y superioridad hacen que alguien acepte ser violentado, ya que se normaliza este comportamiento. Como señaló un participante: "hay circunstancias relacionadas al entorno y al rol de género... (el hombre) como proveedor y figura de autoridad que repercuten en emociones y conductas" o bien "porque se enseñan conductas violentas de alguna forma desencadenando las posiciones de sometimiento y victimización".

\section{b) Consecuencias de la violencia.}

Con respecto a las consecuencias de la violencia en las personas que la reciben, la mayoría de los participantes respondió que es 
el daño psicológico e incluye sentimientos de tristeza, miedo, baja autoestima, inseguridad y desconfianza (ver tabla 2). También reconocieron que una consecuencia importante puede ser volverse agresores y normalizar esta forma de relación. Al respecto, una participante comentó: "si permites que te golpeen una vez te van a golpear muchísimas... las veces que la persona quiera". La mayoría de las mujeres señaló que puede ocasionar la muerte de la víctima. Los hombres mencionaron el daño físico, la disminución de productividad, ya sea en el trabajo o escuela; señalaron, también, que pueden ocurrir demandas legales, o bien, separación en caso de matrimonios.

\section{c) Prevención de la violencia.}

En lo que respecta a las medidas para prevenir la violencia en la vida cotidiana, hombres y mujeres coincidieron al mencionar la importancia de la información, por ejemplo, publicitar refiriéndose a la importancia de dar pláticas y talleres: "asistir a las pláticas que te dan en la escuela, cuando estás en la prepa y... es así... de que la violencia es mala y cosas así”. Los hombres mencionaron un mayor número de medidas de prevención en comparación con las mujeres, como: "no exponerse a situaciones de riesgo, la investigación sobre lo que genera esta problemática”. También propusieron una serie de acciones, como las manifestaciones, las marchas, donde un grupo unido trata de generar conciencia: "a partir de un grupo unido, que evidencia las repercusiones que este caso trae la... la violencia consigo, pues generar conciencia, y mostrar que juntos se lucha para acabar con la problemática...”. Ambos señalaron la importancia del aspecto legal, en términos de que las personas deben conocer sus derechos o las acciones legales que se pueden ejercer,

Tabla 2

Categorías de respuesta a la pregunta, ¿cuáles son las consecuencias de la violencia en las personas que la reciben?

\begin{tabular}{lccc}
\hline & Total & Hombres & Mujeres \\
\hline Volverse agresor & 6 & 2 & 4 \\
Daño psicológico & 16 & 8 & 8 \\
Muerte & 8 & 1 & 7 \\
Disminución en la productividad & 7 & 6 & 6 \\
Demanda en la separación & 1 & 1 & 1 \\
Relación tóxica & 1 & 0 & 1 \\
Costo emocional & 1 & 2 & 2 \\
Normalización de la violencia & 4 & 3 & 0 \\
Daño físico & 3 & 3 & 1 \\
\hline
\end{tabular}


el uso de la publicidad mediática, fomentar la comunicación, y buscar información por iniciativa propia sobre el tema.

\section{Discusión}

Aunque en el mundo actual la violencia es vivida por innumerables personas, también es cierto que muchas formas de violencia son en apariencia invisibles debido a su naturalización como formas de relación. En este estudio, el interés fue explorar la percepción que tienen hombres y mujeres de la ciudad de Mérida sobre el significado de violencia, caracteristicas de agresores y víctimas, causas de la violencia, consecuencias y prevención. Los resultados obtenidos sugieren que hay cierto conocimiento sobre aquellos comportamientos que son violentos, aunque de primera instancia se refieren al daño físico, que puede ocurrir en diversos ámbitos, siendo el más frecuente al interior de la familia, aunque se reconocen otros contextos. También se evidencia cierto conocimiento sobre el papel del contexto social, a través de dos mecanismos: uno la socialización en la familia de modos de relación violentos que se reproducen ulteriormente, o bien, la promoción de formas de solucionar problemas violentos; el otro factor de influencia es la relación de poder establecida entre las personas, lo cual lleva a este ejercicio violento. Las respuestas coinciden con lo que afirma García Canal, (2006) sobre la palabra violencia, a saber, su vinculación con diversas ideas como agresión y agresividad, daño, y muerte.
Respecto a los agresores, se les describe con numerosas características psicológicas negativas, e incluso se adjudica la conducta violenta a desórdenes mentales. En este sentido, se privilegian como causas de la conducta violenta los factores o disposiciones internas, de personalidad (como baja autoestima, falta de seguridad, emociones negativas, sentimientos de superioridad, temperamento) y se devalúa o minimiza el papel del ambiente social, ya que una minoría de participantes reconoce el papel de la crianza de roles de género sexistas que otorgan el poder a los hombres sobre las mujeres. En este sentido, se vislumbra cierto reconocimiento explícito sobre la violencia y su relación estrecha con la expresión de poder, como señala Arendt (2005). Este pobre reconocimiento de la violencia como algo aprendido, señalamos que tiene implicaciones graves, pues esta consideración implicaría que poco puede hacerse para eliminarla al considerarla parte de la naturaleza humana, así como una respuesta "normal" ante diversas situaciones interpersonales. Tampoco se reconocieron otros tipos de violencia como la autoinflingida y colectiva.

En cuanto a las personas que consideran susceptibles de recibir violencia (víctimas), una mayoría respondió que todos podrían ser víctimas, sin embargo, también señaló como más propensas a las mujeres, aunque nunca se menciona el feminicidio (la forma extrema de la violencia de género), y en segundo término a los niños. Resaltaron como factores predisponentes 
ciertas características psicológicas (personas inseguras, calladas, con baja autoestima, diferentes), condiciones sociales (de bajos recursos, grupos minoritarios, personas solas) y grupo de edad (niños, mujeres, adultos mayores). En general, aún se aprecia en estas respuestas un pobre reconocimiento acerca de otros grupos sociales que sufren violencia cotidianamente, como la dirigida a indígenas, personas con capacidades diferentes, la violencia en el noviazgo, en los contextos laborales (mobbing), o en la calle (acoso callejero), lo cual parece una invisibilización (y normalización) de ciertas formas de violencia cotidiana. Como señala Kristinsdóttir (2015), “detrás de la normalización de la violencia se encuentran los discursos, es decir, saberes y verdades representativos de los contextos históricos y culturales de cualquier lugar, que producen las normas y las percepciones existentes en una sociedad" (p. 103).

Si se analizan las atribuciones o causas de la conducta violenta, a partir del modelo ecológico propuesto por la OMS (2002), se observa que los participantes depositan la causa de la conducta violenta en dos factores: individuales (características de personalidad ya citadas), y factores externos, relacionales, relativos a las experiencias y aprendizajes en la familia. Otros dos contextos explicativos no fueron referidos en las respuestas: los contextos comunitarios como la escuela, vecindario, lugares de trabajo, la pobreza, densidad poblacional, presencia de alcoholismo, entre muchos otros.
Por último, están los factores relativos a la estructura de la sociedad (políticas de salud, económicas, educativas) que mantienen las desigualdades sociales. Por ello, se aprecia una visión simplista e inacabada de esta grave problemática, ya que los estudios muestran que las probabilidades de ser víctima o agresor son similares para ambos sexos, y que variables como la exposición a la violencia en la niñez, actitudes favorables a la violencia, bajo nivel educativo y consumo de alcohol son factores asociados a esta problemática (Bair-Merritt, et al., 2010; Fiestas, Rojas, Gushiken \& Gozzer, 2012; Muñoz, Gutiérrez, Arango \& Guerrero, 2004), condiciones que no son reconocidas por los participantes

Sobre las consecuencias, para todos los participantes fue evidente el daño que la violencia genera en las personas, tanto en el ámbito físico como psicológico y resaltaron que quien sufre de violencia también puede responder con violencia hacia otras personas. Las mujeres fueron quienes mencionaron que la violencia puede ocasionar la muerte y los hombres señalaron el daño psicológico, así como consecuencias legales o separación de la pareja. Como puede apreciarse, los resultados sugieren que hay una mirada diferente hacia las consecuencias en función de si el participante es hombre o mujer. Sin embargo, ambos se concretaron a referir unas pocas consecuencias a nivel individual, sin considerar otras formas de violencia a nivel comunitario, además de que no se encontraron respuestas que reflejaran una 
reflexión crítica y profunda sobre los alcances sociales de esta problemática

Finalmente, en cuanto a la prevención, se evidencia un claro desconocimiento en este tema, debido a respuestas como "no exponerse a situaciones de riesgo", lo cual implica que quien sufre de violencia hace algo para merecerla, o al menos se encuentra en situaciones en las que no debería estar. No se mencionaron más que someramente situaciones de desigualdad de género como factores explicativos. Tanto hombres como mujeres mostraron bastante desconocimiento sobre la prevención, en general, señalaron que a través de pláticas, dar información o la publicidad puede prevenirse esta problemática. No se menciona el papel del contexto educativo escolar y comunitario, de los medios de comunicación y las nuevas tecnologías como recursos para "desestructurar aquellas relaciones y actividades que reproducen la violencia" (Valdez-Santiago et al., 2013, p. 260). En conclusión, este estudio ha evidenciado que los participantes poseen conocimientos limitados sobre la violencia interpersonal, aunque conocen diferentes expresiones de violencia y algunos contextos en los que se manifiesta. Se evidencian también muchas imprecisiones o mitos sobre la causa de la violencia y las características de los agresores y víctimas, así como sobre la posibilidad de prevenirla. De ahí la importancia de generar conocimiento propio a partir del cual reforzar en los jóvenes la sensibilización contra la violencia en sus diversas manifestaciones a través de distintas estrategias. Por ultimo, es importante recalcar la importancia de hacer más estudios en esta línea, dada la naturalización e invisibilización de la violencia y sus diversas manifestaciones y consecuencias entre los jóvenes, considerando en futuros estudios diversos niveles educativos y grupos de edad, así como población urbana y rural, variables que no son tomadas en cuenta en este estudio.

\section{Referencias}

Arendt, H. (2005). Sobre la violencia. Madrid: Alianza Editorial.

Bair-Merrit, M., Crowne, S., Thompson, D., Sibinga, E., Trent, M. y Campbell, J. (2010). Why do women use intimate partner violence? A systematic review of women's motivations. Trauma Violence Abuse, 11(4), 178-89.

Briceño-León, R. (2008). La violencia homicida en América Latina. América Latina Hoy, 50, 103-116.

Cerezo, F. y Ato, M. (2010). Estatus social, género, clima del aula $y$ bullying entre estudiantes adolescentes. Anales de Psicología, 26(1), 137-144. doi:10.6018/92131

ENDIREH. (2016). Encuesta Nacional sobre la Dinámica de las Relaciones en los hogares. México: INEGI. Recuperado de http://www.beta.inegi.org.mx/contenidos/ programas/endireh/2016/doc/endireh2016_ presentacion_ejecutiva.pdf 
Esquivel, J. A, Jiménez-Bautista, F. y EsquivelSánchez, J. A. (2009). La relación entre conflictos y poder. Revista de Pazy Conflictos, 2, 6-23.

Fiestas, F., Rojas, R., Gushiken, A. y Gozzer, E. (2012). ¿Quién es la víctima y quién el agresor en la violencia física entre parejas? Estudio epidemiológico en siete ciudades del Perú. Revista Peruana de Medicina Experimental y Salud Publica, 29(1), 44-52. Recuperado de http://www.ins.gob.pe/insvirtual/images/ artrevista/pdf/rpmesp2012.v29.n1.a07.pdf

Foucalt, M. (1999). Estrategias de poder. Barcelona: Paidós.

Freisthler, B., Merritt, D. H. y LaScala, E. A. (2006). Understanding the Ecology of Child Maltreatment: A Review of the Literature and Directions for Future Research. Child Maltreatment, 11(3), 263-280.

Frías, S. y Castro, R. (2011). Socialización y violencia: Desarrollo de un modelo de extensión de la violencia interpersonal a lo largo de la vida. Estudios Sociológicos, 26(86), 497-550. doi: 10.2307/23034045

Fuentes, M. C., Martínez, I. y Navarro, F. (2015).

Spanish Parenting Styles and Adolescence Bullying. En F. García (Ed.), Parenting: cultural influences and impact on childhood health and well-being (Cap. 9). Hauppauge, NY: Nova Science Publishers, Inc.

García-Canal, M. I. (2006). Poder, violencia y palabras. Tramas, 25, 113-128. Recuperado de http://132.248.9.34/hevila/ TramasMexicoDF/2005/no25/5.pdf
Gracia, E. y Herrero, J. (2008). Is It Considered Violence? The Acceptability of Physical Punishment of Children in Europe. Journal of Marriage and Family, 70(1), 210-217.

Hernández-Sampieri, R., Fernández-Collado, C. y Baptista-Lucio, P. (2014). Metodología de la investigación. México: McGrall-Hill.

INEGI. (2007). Encuesta Nacional sobre Violencia en el Noviazgo. Informe operativo. México: SEP-IMJ. Recuperado de https://www.imjuventud.gob.mx/imgs/ uploads/ENVINOV_2007_-_Resultados_ Generales_2008.pdf

INEGI. (2017). Datos preliminares revelan que en 2017 se registraron 31 mil 174 homicidios. Comunicado de prensa. Recuperado dehttps:// aristeguinoticias.com/3007/mexico/2017-elano-mas-violento-de-la-historia-del-pais-seregistraron-31-mil-174-homicidios-inegi/

Informe sobre la Situación Mundial de la Prevención de Violencia. (2014). Recuperado de https://apps.who.int/iris/ bitstream/handle/10665/145089/WHO_ $\mathrm{NMH}$ _?sequence $=1$

Jiménez-Bautista, F. (2012). Conocer para comprender la violencia: Origen, causas y realidad. Convergencia. Revista de Ciencias Sociales, 19(58), 13-52.

Kristinsdóttir, R. S. (2015). Cultura de violencia: Normalización de la violencia de género en Guatemala. Recuperado de https:// gupea.ub.gu.se/bitstream/2077/38420/1/ gupea_2077_38420_1.pdf 
Maliandi, R. (2010). Ética Convergente. Fenomenología de la conflictividad. Buenos Aires: Las Cuarenta. Recuperado de http:// aaieticas.org/revista/index.php/cde/article/ view/23/48

Miller, T. R., Cohen, M. A. y Rossman, S. B. (1993). Victim costs of violent crime and resulting injuries. Health affairs, 12(4), 186197. doi: 10.1377/hlthaff.12.4.186

Molnar, B. E., Buka, S. L., Brennan, R. T., Holton, J. K. y Earls, F. (2003). A Multilevel Study of Neighborhoods and Parent-toChild Physical Aggression: Results from the Project on Human Development in Chicago Neighborhoods. Child Maltreatment, 8(2), 84-97.

Muñoz, E., Gutiérrez, M., Arango, O. y Guerrero, R. (2004). Creencias, Actitudes y Prácticas sobre Violencia en Bogotá, Cali y Medellín. Línea de Base. Santiago de Cali: Ministerio de Protección Social, Universidad del Valle, Instituto CISALVA. Recuperado de http:// grupocisalva.univalle.edu.co/publicaciones/ documentos/linea_de_base.pdf

Oliver, W., Kuhns, L. y Pomeranz, E. (2006). Family Structure and Child Abuse. Clinical Pediatrics, 45(2), 111-118. doi: 10.1177/000992280604500201

OMS. (2002). Informe mundial sobre violencia y salud: Resumen. Whashington: Organización Mundial de la Salud. Recuperado de https:// www.who.int/violence_injury_prevention/ violence/world_report/es/summary_es.pdf
OMS. (2005). Estudio multipaís de la OMS sobre salud de la mujer y violencia doméstica. Primeros resultados sobre prevalencia, eventos relativos a la salud y respuesta de las mujeres a dicha violencia: resumen del informe. Ginebra: Ediciones de la OMS. Recuperado de http://www.who.int/ gender/violence/who_multicountry_study/ summary_report/summaryreportSpanishlow. pdf

Organización Panamericana de la Salud. (2002). Informe Mundial sobre la Violencia y la Salud. Washington, DC: Organización Mundial de la Salud. Recuperado de https:// www.who.int/violence_injury_prevention/ violence/world_report/es/summary_es.pdf

Osorio, S. (2012). Conflicto, Violencia y Paz: Un acercamiento científico, filosófico y bioético. Revista Latinoamericana de Bioética, 12(2), 052-069. Recuperado de http://www. redalyc. org/articulo.oa?id=127025833006

Paredes-Guerrero, L., Llanes-Salazar, R., Torres-Salas, N. y España-Paredes, A. (2016). La violencia de género contra las mujeres en Yucatán. LiminaR. Estudios Sociales y Humanísticos, 14(2), 45-56.

Pegalajar, P. M. C. (2018). Análisis del estilo de gestión del conflicto interpersonal en estudiantes universitarios. Revista Iberoamericana de Educación, 77(2), 9-30.

Quiroga, S. y Cryan, G. (2007). Manifestaciones de la violencia en adolescentes de alto riesgo. XIV Jornadas de Investigación y Tercer 
Encuentro de Investigadores en Psicología del Mercosur. Buenos Aires: Facultad de Psicología. Universidad de Buenos Aires. Recuperado de https://www.aacademica. org/000-073/145

Romero-Abrio, A., Musitu, G., CallejasJerónimo, J. E., Sánchez-Sosa, J. C. y Villarreal-González, M. E. (2018). Factores predictores de la violencia relacional en la adolescencia. Liberabit, 24(1), 29-43. Recuperado de https://doi.org/10.24265/ liberabit.2018.v24n1.03

Rubio, C. J., Chávez, E. M. y Rodríguez, R. H. (2017). Significados, causas y efectos de la violencia social entre la juventud en Monterrey, Nuevo León, México. Revista Sociedad y Economía, 32, 86-106.

Silva, G. (2008). La teoría del conflicto. Un marco teórico necesario. Prolegómenos. Derechos y Valores, 11(22), 29-43. Recuperado de http:// www.redalyc.org/articulo. oa?id=87602203 Suárez-Relinque, C., Del Moral-Arroyo, G., Martínez-Ferrer, B. y Musitu, G. (2015). Spanish parental socialization styles, school and social to parent violence. En F. García (Ed.), Parenting: cultural influences and impact on childhood health and well-being (pp. 166-177). Hauppauge, NY: Nova Science Publishers, Inc.

Tajima, E. (2002). Risk Factors for Violence Against Children: Comparing Homes With and Without Wife Abuse. Journal of Interpersonal Violence, 17(2), 122-149.
Terceño, S. C. (2017). Estilos de socialization parental y violencia filioparental en la adolescencia (Tesis Doctoral). España: Universidad de Sevilla. Recuperado de https://www.uv.es/lisis/otras-publica/tcandelaria.pdf

Unnithan, N. y Whitt, H. (1992). Inequality, economic development and lethal violence: a crossnational analysis of suicide and homicide. International Journal of Comparative Sociology, 33(3-4), 182-196. doi: 10.1163/002071592X00239

Valdez-Santiago, R., Hidalgo-Solórzano, E., Mojarro-Íñiguez, M., Rivera-Rivera, L. y Ramos-Lira, L. (2013). Violencia interpersonal en jóvenes mexicanos $\mathrm{y}$ oportunidades de prevención. Salud Pública de México, 55(2), 259-266.

WHO, Global Health Observatory. (2016). World Health Organization. Recuperado de https:// apps.who.int/iris/bitstream/handle/10665/ 112738/9789240692671_eng.pdf;jsessionid $=77 \mathrm{~F} 1482 \mathrm{C} 20547 \mathrm{~B} 60 \mathrm{C} 646503 \mathrm{DB} 14 \mathrm{D} 3 \mathrm{EE}$ 5 ? sequence $=1$

Recibido: 04/04/2019

Revisado: 25/06/2019

Aceptado: 03/07/2019 